\title{
Modulation of the Thermochemical Stability and Adsorptive Properties of MOF-808 by the Selection of Non-structural Ligands
}

\author{
Erlend Aunan, ${ }^{\S}$ Christopher W. Affolter, ${ }^{\S}$ Unni Olsbye, and Karl Petter Lillerud*
}

Cite This: Chem. Mater. 2021, 33, 1471-1476

Read Online

ABSTRACT: Metal-organic frameworks (MOFs) have been heavily researched due to their porous and crystalline nature. Because of this, MOFs are enticing materials for fields as diverse as gas separation and purification, water harvesting, and heterogeneous catalysis. Within most potential applications, it is critical for the material to remain unchanged when operating over prolonged periods and/or at elevated temperatures. Therefore, thermal stability is crucial in order to utilize MOFs industrially. In this article, we present a method to thermally stabilize MOF-808 by employing post-synthetic functionalization to incorporate benzoate moieties into its framework. We further describe how this incorporation changes the MOFs' physicochemical properties (i.e., surface area, gas-adsorptive behavior, and crystallographic

structure). The materials described herein are well characterized and provide novel options for the design of stable catalysts and adsorbents for future technologies.

$\mathrm{M}$ etal-organic frameworks (MOFs) have rapidly expanded as a field of research over the past 30 years due to the exceedingly porous and crystalline nature of these coordination polymers. MOFs have also been commended for their structural predictability, which has led to the development of reticular chemistry as a field. ${ }^{1,2}$ The highly defined crystallinity of MOFs, coupled with the level of synthetic control that they afford and the possibility to tailor the surroundings of the functional groups present in the framework, are remarkable-elevating their potential as superior platforms for heterogeneous catalysts.

In order to prevail as a heterogeneous catalyst, or as a catalyst support, it is vital for the MOF to remain unchanged after prolonged exposure to elevated temperatures. Moreover, it is important to consider the feasibility of synthesizing or functionalizing an MOF to be used in industrial processes. The synthesis must be affordable, robust, scalable, and preferably green. Some MOFs provide options that fulfill many of these requirements. Zirconium-based MOFs (Zr-MOFs) in particular, primarily due to their thermal, chemical, and physical stability, appear to be promising candidates.

In this article, we investigate MOF-808, which could be ideal as a scaffold material or as a heterogeneous catalyst itself. In addition to being synthesizable using a green, water-based protocol, MOF- 808 also maintains great stability and is highly amenable to post-synthetic functionalization due to the presence of non-structural sites on its unique six-coordinated clusters. Herein, we report a novel derivative of MOF-808, in which incorporation of benzoate moieties into the available

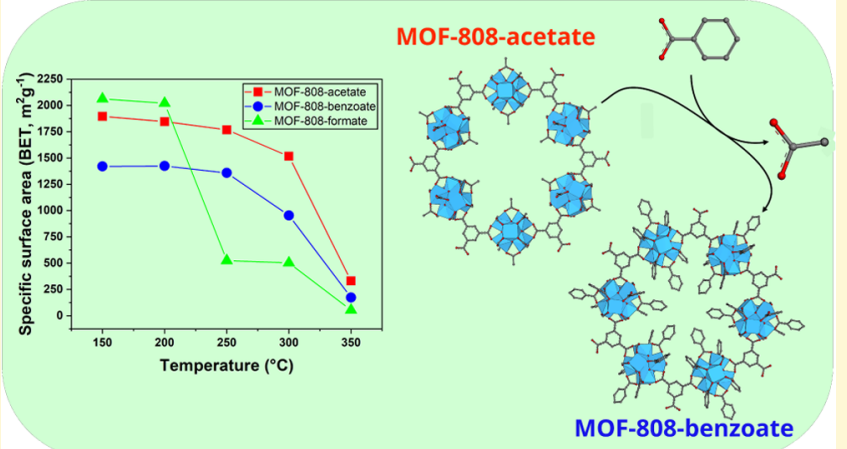

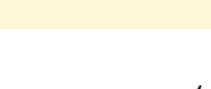

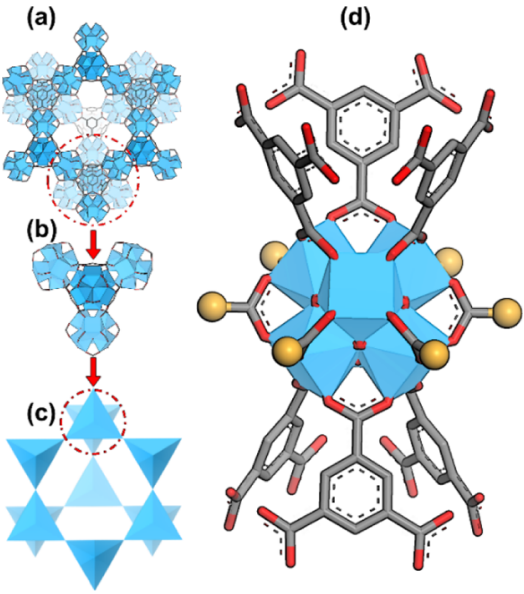

Figure 1. Schematic overview of MOF-808 (a), its tetrahedral tertiary building unit (TBU) (b), its simplified network (spn, shown as dia-a, c), and an illustration of the secondary building unit (SBU) showing where the non-structural ligands are geometrically located, indicated by orange spheres (d). Hydrogen atoms are omitted for clarity. Carbon: black, oxygen: red, zirconium: blue polyhedra, and nonstructural ligand: orange.

Received: December 18, 2020

Revised: January 26, 2021

Published: February 8, 2021 
non-structural sites of the framework results in a material, MOF-808-benzoate, with unique physicochemical properties and exceptional stability.

\section{BACKGROUND}

Interest in $\mathrm{Zr}$-MOFs began with the discovery of UiO-66 $\left(\mathrm{Zr}_{6} \mathrm{O}_{4}\left(\mathrm{OH}_{4}\right)(\mathrm{BDC})_{6}\right)$ published by Cavka et al. in 2008, ${ }^{3}$ and since that time, they have remained a benchmark standard for new MOFs because of their groundbreaking stability. This stability is derived from their fcu structure, consisting of each $\left[\mathrm{Zr}_{6}\left(\mu_{3}-\mathrm{O}\right)_{4}\left(\mu_{3}-\mathrm{OH}\right)_{4}\right]^{12+}$ unit fully connected by 12 ditopic linkers (terephthalic acid, $\mathrm{H}_{2} \mathrm{BDC}$ ). This provides a saturated, 12-coordinated SBU (a deviation from this 12-coordination can be referred to as an unsaturated SBU, where a structural vacancy is present). However, although a fully 12 -coordinated Zr-SBU provides stability, an unsaturated SBU can often be desired since a lesser connected cluster will have more accessible metal sites. Hence, several MOFs have recently shown interesting catalytic properties due to structural vacancies. ${ }^{4,5}$ Such vacancies are achieved by structural defectivity (e.g., missing linkers) or by the MOF's inherent topology. Examples of $\mathrm{Zr}$-MOFs with inherent topological vacancies are DUT-69 (10-coordinated), ${ }^{6}$ NU-1000 (8coordinated), ${ }^{7}$ MOF-808 (6 coordinated), ${ }^{8}$ and NU-1400 (4-coordinated). ${ }^{9}$ These structural vacancies, in addition to being potentially catalytically active, provide the possibility of post-synthetic functionalization using monotopic ligands such as monocarboxylates. $\mathrm{Lu}$ et al. recently published an article describing ligand exchange and activation of NU-1000, a method to obtain a homogeneously decorated cluster. ${ }^{10}$ The development of methods to tailor the chemical functionality of MOFs remains a critical area for research involving design and testing of Zr-MOFs in fields such as catalysis, gas storage, gas separation and purification, drug delivery, and fluorescent sensing. ${ }^{11}$

General Properties and Uses. MOF-808 is a zirconiumbased MOF first described by the Yaghi group from the University of Berkeley in $2014 .{ }^{8}$ Although sharing the same ZrSBU as UiO-66, the crystal structure of MOF-808 differs greatly, as it consists of only six tritopic linkers (trimesic acid, $\mathrm{H}_{3} \mathrm{BTC}$ ) coordinating each SBU. This topology (spn) requires an additional six monotopic ligands to be located in an equatorial belt around the SBU to provide charge compensation. These six non-structural ligands (depicted as L), which have been described in the literature to be a mixture of formate and $\mathrm{H}_{2} \mathrm{O} / \mathrm{OH}^{-}$, are relatively labile and therefore easily exchanged.

MOF-808's topology is best described as corner-sharing tetrahedral subunits consisting of SBUs on the vertices and linkers on the faces (Figure 1). These form a network described with the spn topology or by the augmented diamond net (dia-a) if one considers the tetrahedral TBU. This topology results in two pore shapes: a cage within each tetrahedral TBU and one large adamantane-shaped pore with a pseudohexagonal pore window. ${ }^{8,12}$ As a consequence of this geometry, each ligand is pointing directly into a large pore. The ligand position ensures availability to guest molecules and, combined with the aforementioned lability of ligands, provides a reliable and effective way of introducing new functionality into the MOF via solvent-assisted ligand exchange (SALE). Several procedures have previously been developed to incorporate functionality such as metal-coordinating ligands, ${ }^{13}$ proton conductivity, ${ }^{14}$ and superacidity into MOF-808. ${ }^{15}$
There is a vast difference in the chemical nature of MOF808 depending on the identity of the non-structural ligand. Because of this, we suggest that MOF- 808 should be referred to as a family of materials, rather than as a specific MOF with a specific structure. The most researched member of this family to date is MOF-808-formate, the material which was initially described (Figure 2b). MOF-808-formate was originally
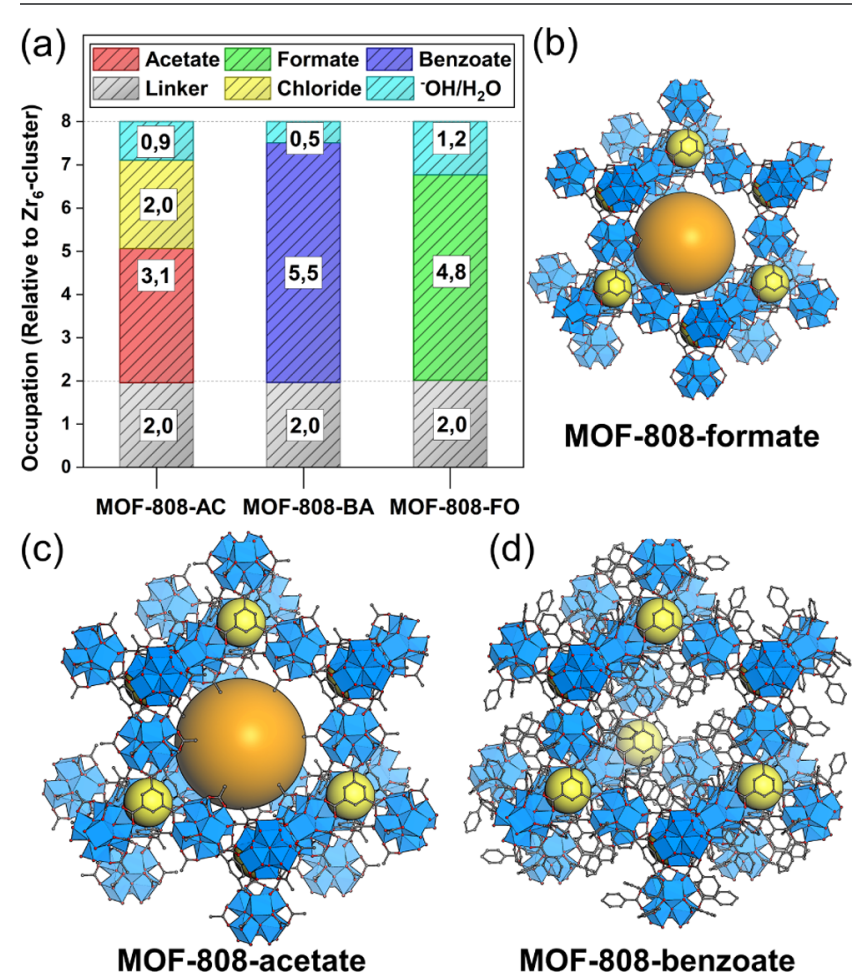

Figure 2. (a) MOF composition of MOF-808-acetate (left), MOF808-benzoate (middle), and MOF-808-formate (right) shown relative to one $\mathrm{Zr}_{6}$-SBU $\left[\mathrm{Zr}_{6} \mathrm{O}_{4}\left(\mathrm{OH}_{4}\right)^{12+}\right]$. Illustration of (b) MOF-808formate, (c) MOF-808-acetate, and (d) MOF-808-benzoate. Orange and yellow spheres indicate empty space. Hydrogen atoms are omitted for clarity. Carbon: black, oxygen: red, and zirconium: blue polyhedra.

investigated as a potential water-adsorbing agent. ${ }^{8}$ Although outcompeted by other Zr-MOFs under very low humidity conditions, it exhibited the second highest total water uptake of the MOFs tested. Various MOF-808s have also shown promising results in the adsorption of highly toxic chemical species such as organoarsenic acids, ${ }^{16}$ organophosphorus nerve agents, ${ }^{17}$ and heavy metal ions such as $\mathrm{Hg}(\mathrm{II}), \mathrm{Cd}(\mathrm{II})$, and $\mathrm{Pb}$ (II). ${ }^{18,19}$ The Farha group have shown that in addition to its adsorptive capability, activated MOF-808 $\left(\mathrm{Zr}_{6}\left(\mu_{3}-\mathrm{O}\right)_{4}\left(\mu_{3}-\right.\right.$ $\left.\mathrm{OH})_{4}(\mathrm{BTC})_{2}(\mathrm{OH})_{6}\left(\mathrm{H}_{2} \mathrm{O}\right)_{6}\right)$ displays the highest reported catalytic hydrolysis rate of DMNP, a nerve agent simulant. ${ }^{20}$ These diverse examples of applications for a single member of the MOF-808 family clearly demonstrate the great potential for ligand exchange at non-structural sites to produce novel MOF808 derivatives with as yet undiscovered properties.

\section{EXPERIMENTAL SECTION}

Synthesis. MOF-808-formate is typically synthesized using the original protocol published in 2014 by Furukawa et al., ${ }^{8}$ or slightly modified versions of it. These protocols generally yield MOF-808formate in microcrystalline form of excellent quality. However, these protocols involve the usage of toxic and flammable $\mathrm{N}, \mathrm{N}$ - 


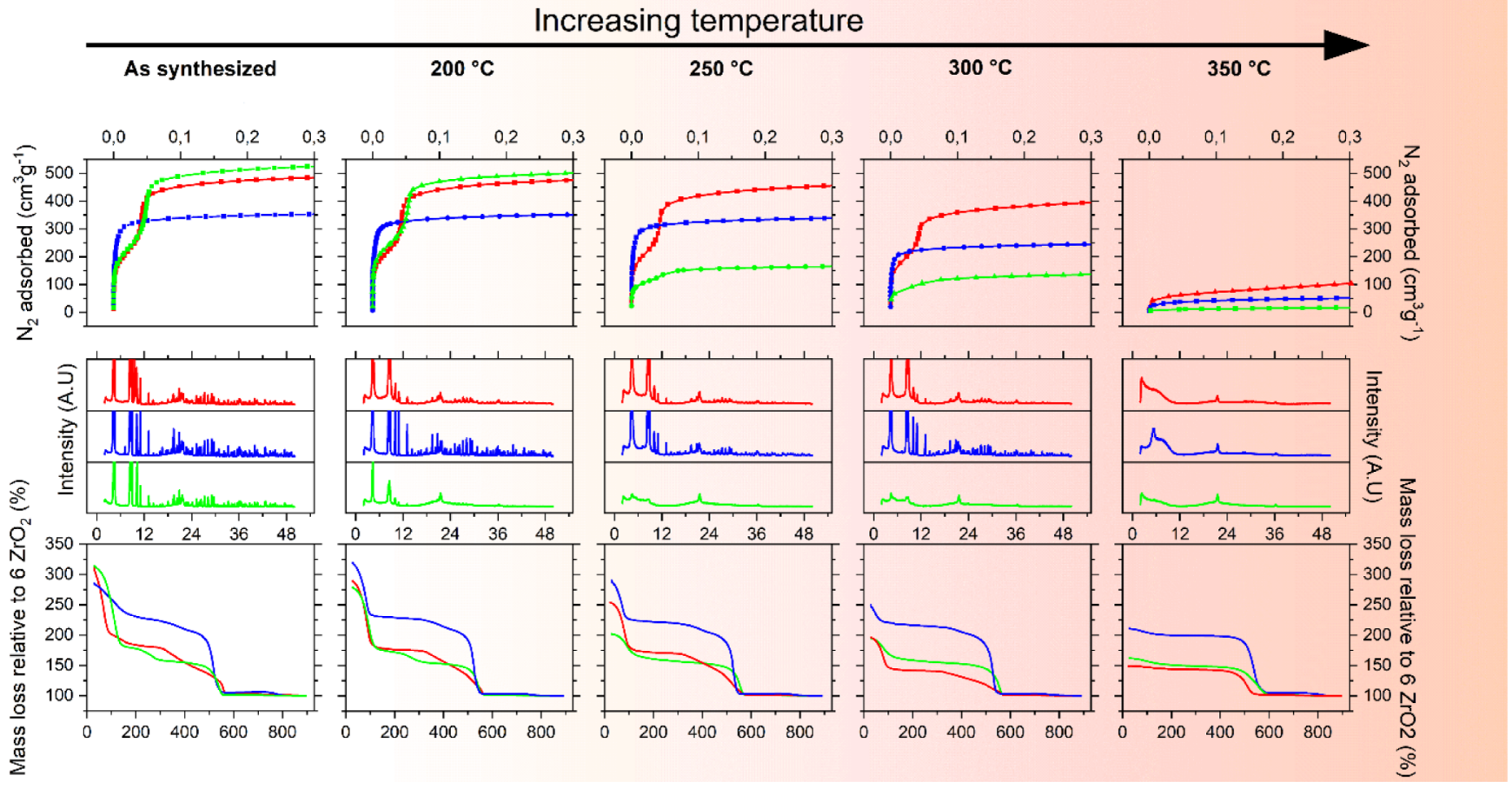

Figure 3. Heat stability assessment of MOF-808-X, where $\mathrm{X}$ is acetate (red), benzoate (blue), or formate (green), using nitrogen adsorption isotherms [top, $x$-axis: relative pressure $\left(p / p_{0}\right)$ ], powder X-ray diffractograms [middle, $x$-axis: diffraction angle $\left(2 \theta, \mathrm{Cu}_{\mathrm{K} \alpha}\right)$ ], and thermogravimetric analysis (TGA) [bottom, $x$-axis: temperature $\left({ }^{\circ} \mathrm{C}\right)$ ]. The temperature is increasing from left to right. Each sample of MOF was heated and kept at the denoted temperature for $16 \mathrm{~h}$.
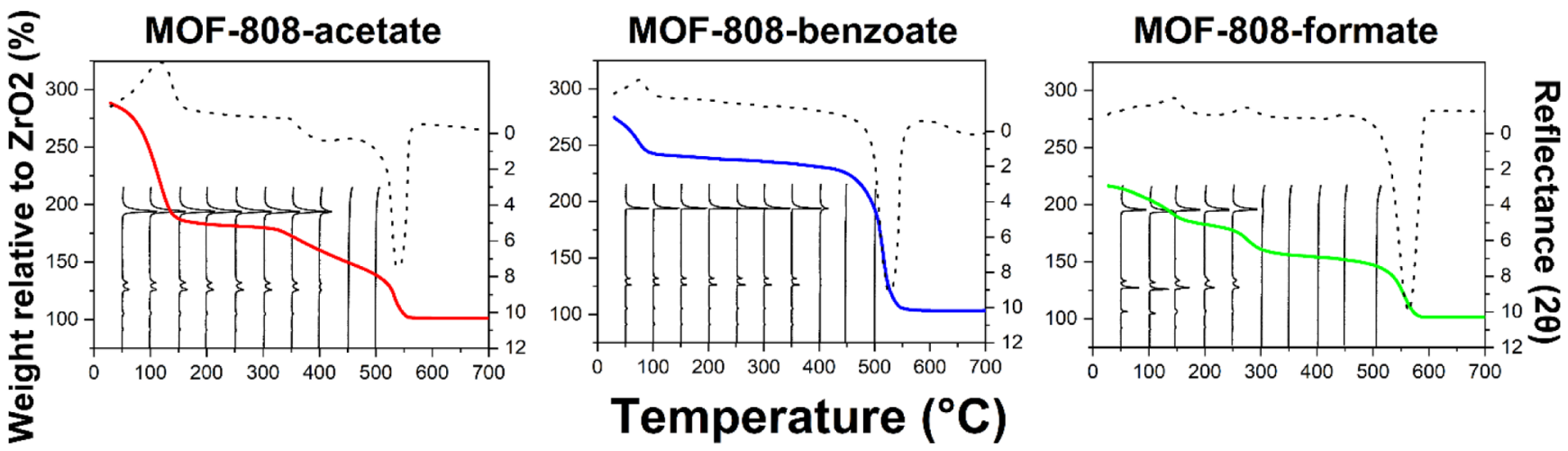

Figure 4. Temperature-dependent PXRD of MOF-808-benzoate (left), MOF-808-acetate (middle), and MOF-808-formate (left) plotted against their respective thermogravimetric curves (colored lines, differential scanning calorimetry (DSC) signal as dotted line, where a negative signal is indicating exothermicity, unit: $\mathrm{mW}$ ). The vertically arranged diffractograms are lined up with the TG curve's $x$-axis (temperature) and indicate the structure collapse in agreement with the weight loss curve. The heating rate in both the TG and X-ray diffraction experiments was $5 \mathrm{~K}^{\text {min }}{ }^{-1}$.

dimethylformamide (DMF), as well as sealed glass vessels, very dilute reaction mixtures, and long reaction times. ${ }^{8,15}$ Therefore, in this work, we applied a more efficient procedure which is a modified version of the water-based protocol published by Reinsch et al. ${ }^{21}$ that employs a concentrated synthesis and requires a shorter reaction time. Using this protocol, we were able to synthesize high-quality MOF-808-formate as well as MOF-808-acetate (Figure 2c) at a large scale $(10-1000 \mathrm{~g}$ ).

The products yielded by the water-based syntheses differ from the DMF-based protocol in two major ways. First, the material incorporates chloride anions, occupying roughly two out of the six open coordination sites. However, the chloride can be exchanged for formate or acetate using aqueous solutions of their respective sodium salts. Once chloride has been exchanged, the characteristics of the products appear to be otherwise indistinguishable from the DMFbased material, in terms of X-ray diffraction patterns, surface areas, and approximate chemical composition. Second, the crystallite size of the product from the water-based protocol is significantly larger than the ones from the original protocol. Although, this is in many cases a benefit, as it aids general handling of the material, the size could limit the applicability of the method for synthesizing materials for applications in which diffusion paths into the material must be minimal.

Ligand Exchange. By post-synthetic SALE, we were able to synthesize the novel material MOF-808-benzoate with the approximate chemical formula $\mathrm{Zr}_{6} \mathrm{O}_{4}(\mathrm{OH})_{4}(\mathrm{BTC})_{2}\left(\mathrm{Ph}-\mathrm{CO}_{2}\right)_{6}$. Benzoate incorporation was achieved by dispersing MOF-808-acetate (as synthesized, with chloride) in a saturated aqueous solution of benzoic acid at elevated temperatures $\left(60{ }^{\circ} \mathrm{C}\right.$ ) for between 16 and $20 \mathrm{~h}$ (see the Supporting Information for detailed synthetic conditions). The resulting powder, isolated either by centrifugation or by filtration, was then washed with warm water, followed by acetone, and dried in an oven at $120{ }^{\circ} \mathrm{C}$ overnight, yielding MOF-808-benzoate (Figure 2d). 


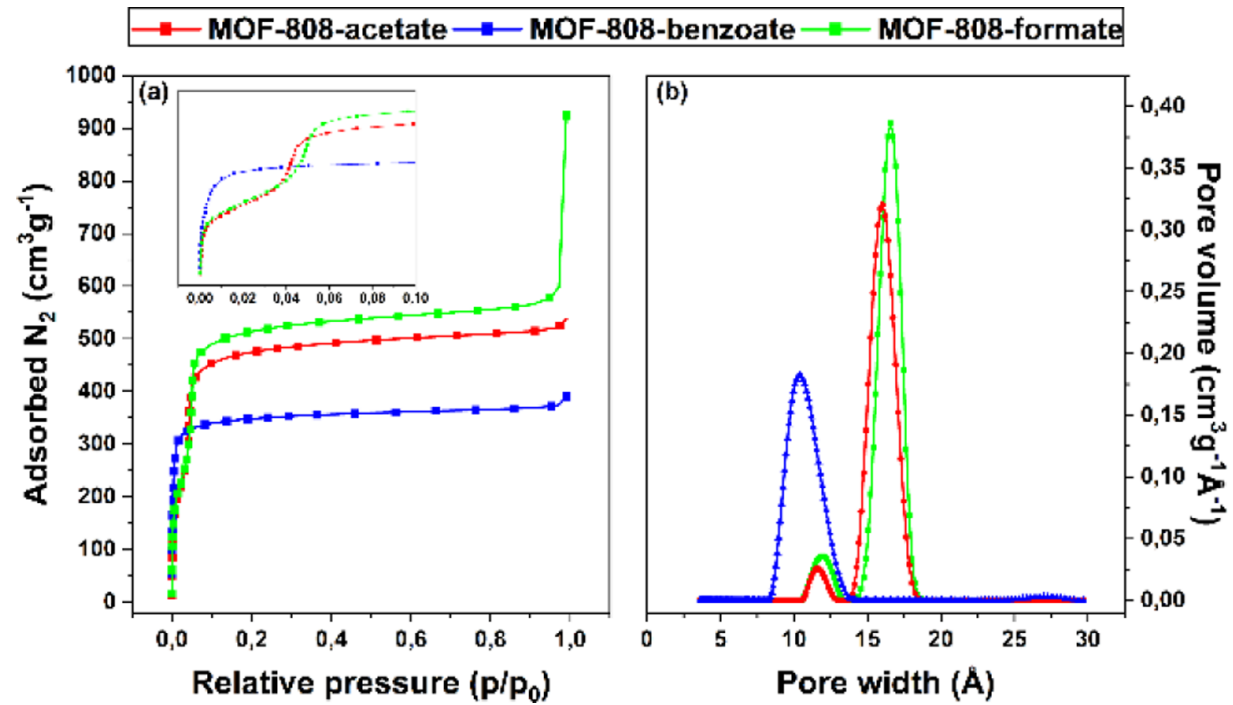

Figure 5. (a) Nitrogen adsorption isotherm (a, left) and pore size distribution (b, right) for MOF-808-acetate (red), benzoate (blue), and formate (green).

\section{RESULTS}

The shape and color of the isolated materials were identical when compared to the parent material. The obtained powder $\mathrm{X}$-ray diffracton patterns indicated no significant change, apart from a unit cell expansion of approximately $0.2 \%$ (Supporting Information SI-1). The progression of ligand exchange was evaluated by using a combination of TGA, proton nuclear magnetic resonance $\left({ }^{1} \mathrm{H} \mathrm{NMR}\right)$, and energy-dispersive X-ray spectroscopy. The results from these techniques were then combined and the amounts of linker, ligand, and chloride, reported relative to one zirconium SBU $\left(\left[\mathrm{Zr}_{6} \mathrm{O}_{4}\left(\mathrm{OH}_{4}\right)\right]^{12+}\right)$, were obtained. The combination of these techniques provides an overview of the MOFs' approximate composition, as shown in Figure 2a. An almost complete incorporation of benzoate was observed, achieved by displacing acetate as well as chloride.

Long-Term Thermal Stability. In four independent experiments, the samples of MOF-808-formate, MOF-808acetate, and MOF-808-benzoate were heated at different temperatures $\left(200,250,300\right.$, and $\left.350{ }^{\circ} \mathrm{C}\right)$ for $16 \mathrm{~h}$ under an ambient atmosphere. The heated MOFs' porosity and surface area were then measured by $\mathrm{N}_{2}$-adsorption and the crystallinity of the samples was evaluated using PXRD. In addition, thermogravimetry was performed in order to establish how the heat treatment affected the MOFs' overall composition, as well as response to further thermal stress. Our results, presented in Figure 3, indicate that MOF-808-formate loses both porosity and crystallinity at lower temperature than the benzoate and acetate varieties. The decline in the calculated BrunauerEmmett-Teller (BET) surface areas is presented in Figure 6.

Short-Term Thermal Stability. Samples from each of the three materials were analyzed by means of variable temperature PXRD and thermogravimetry. The temperature ramp rates for the two independent experiments were the same $\left(5 \mathrm{~K} \mathrm{~min}^{-1}\right)$, and both performed in synthetic air under normal atmospheric pressure. The results are presented in Figure 4, with the temperature-respective diffractograms superimposed with the thermogravimetric curves.

Nitrogen Adsorption. Nitrogen adsorption isotherms for the three synthesized materials are presented in Figure 5, together with their respective pore size distributions, which

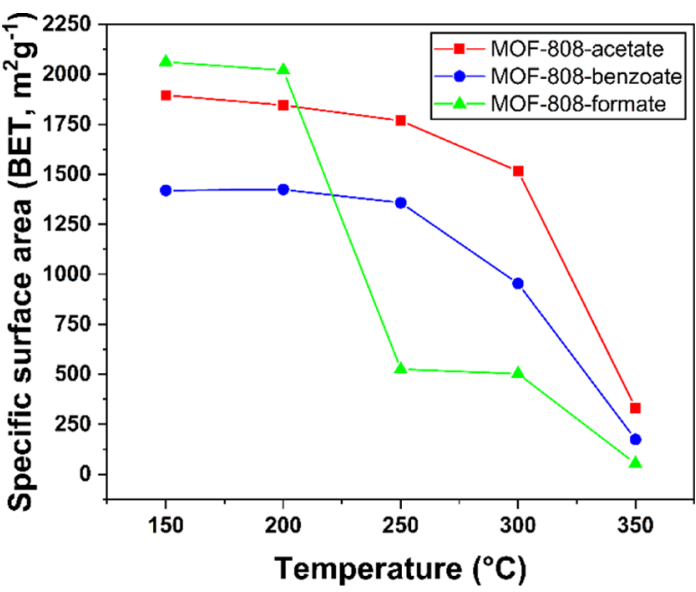

Figure 6. Scatter plot of BET surface areas vs temperature of heattreated samples of MOF-808.

were estimated using non-localized density functional theory (NLDFT)

Adsorption of Other Gasses. Gas sorption experiments ${ }^{22}$ were conducted using carbon dioxide, methane, propylene, propane, isobutylene, and isobutane. Isosteric heats of adsorption were calculated using the Clausius-Clapeyron relation between two sets of isotherms, measured at 273 and $295 \mathrm{~K}$. The adsorption enthalpies are presented in Table 1. The results are also summarized in Supporting Information SI16.

Table 1. Calculated Isosteric Heats of Adsorption (kJ $\mathrm{mol}^{-1}$ ) for MOF-808-Acetate, MOF-808-Benzoate, and MOF-808-Formate Using Isotherm Adsorption Curves at 273 and $295 \mathrm{~K}$

$\begin{array}{lrrrrrc} & \mathrm{CO}_{2} & \mathrm{CH}_{4} & \mathrm{C}_{3} \mathrm{H}_{6} & \mathrm{C}_{3} \mathrm{H}_{8} & \mathrm{C}_{4} \mathrm{H}_{8} & \mathrm{C}_{4} \mathrm{H}_{10} \\ \mathrm{AC} & 21.5 & 9.7 & 25.5 & 24.4 & 29.8 & 29.5 \\ \text { BA } & 24.0 & 16.2 & 30.8 & 31.5 & 36.7 & 36.6 \\ \text { FO } & 20.7 & 12.6 & 25.4 & 25.7 & 30.4 & 29.5\end{array}$




\section{DISCUSSION}

The vast majority of research using MOF-808 that is reported in the literature has been conducted using exclusively the formate analogue. This analogue, as originally described by the Yaghi group, consists of the MOF-808 spn structure decorated by a varying amount of formate anions and water/hydroxide pairs which occupy the structural vacancies on each cluster. However, as seen in the thermostability experiments presented in Figures 3 and 4, the formate analogue exhibits the lowest stability within this suite from the family of MOF-808 materials. We also show that the acetate variant of MOF808 , which has been synthesized with a water-based protocol, can tolerate up to $300{ }^{\circ} \mathrm{C}$ for $16 \mathrm{~h}$ before structural collapse.

The thermally induced structural collapse of MOF-808acetate and MOF-808-benzoate, witnessed by PXRD, occurs contemporarily with the final, exothermic weight loss step observed in the thermogram. Surprisingly for MOF-808formate, the structural collapse happens at a significantly lower temperature than the exothermic step and rather occurs together with a smaller, endothermic step at $250{ }^{\circ} \mathrm{C}$. In order to investigate the nature of this endothermic step, ${ }^{1} \mathrm{H}$ NMR spectroscopy (Supporting Information SI-15) of digested MOF was performed on a sample before and after heating to $250{ }^{\circ} \mathrm{C}$. The spectra reveal a clear absence of formate in the heat-treated sample. Therefore, it is likely that the structural collapse occurs due to decomposition of formate potentially catalyzed by the presence of acidic Zr-metal sites. This observation motivates future studies that lie outside the scope of this work.

The relative instability of MOF-808-formate compared to other members of the MOF- 808 family is in accordance with the results from a similar study by Liang et al. from $2014 .{ }^{23}$ In their study, the authors compared MOF-808-formate with the structural analogues MOF-808-acetate and MOF-808-propionate. These materials were obtained by modifying the original DMF-based synthesis, replacing formic acid with acetic acid or propionic acid, respectively. The authors observed MOF-808acetate exhibiting higher thermal stability when compared to the other two analogues, a finding that is also observed in our study. We therefore propose that MOF-808-acetate, synthesized either in water or in DMF, can be a viable replacement for the formate analogue with few if any downsides. When comparing gas-adsorptive behavior to several gasses, as presented in Table 1, we observed comparable behavior between the acetate and the formate MOF. An example of an application where it may be beneficial to choose the acetate variant over the formate variant would be as a support for a heterogeneous catalyst. We believe that the almost $100{ }^{\circ} \mathrm{C}$ difference of stability is quite significant in terms of reaction conditions for many heterogeneous reactions.

Furthermore, our study also revealed the possibility to drastically modify the MOF's adsorptive behavior by substituting the non-structural ligands with benzoate. Although this substitution results in some reduction in surface area, MOF-808-benzoate exhibits higher affinity to all the tested adsorbates while still displaying the thermal stability advantage of MOF-808-acetate. We believe that this retention of stability is key in order to be trialed as a potential catalyst support.

In addition to the increased heat of adsorptions, the introduction of benzoate changes the behavior of MOF-808 in several other ways. The MOF no longer forms a stable suspension in water, which the parent material MOF-808- acetate does. This change of hydrophobicity is likely due to the addition of the phenyl rings. Nitrogen physisorptive behavior (isothermal at $77 \mathrm{~K}$, Figure 5a) is also drastically changed. The introduction of the bulky phenyl rings is associated with loss in both surface area and capacity. The general shape of the isotherm also changes from the two-step isotherm visible for both MOF-808-acetate and formate into a more typical type-I isotherm with a single step. This is, according to pore-width analysis by NLDFT (Figure $5 b$ ), due to partial filling of the large adamantane-shaped pore. This filling results in a pore width similar to the tetrahedral cage (approximately $10 \AA$ ), creating an apparent monomodal pore size distribution. We find that this change increases the reproducibility of the surface area determination by applying the BET equation to the isotherm. Finally, we argue that the ability to tune the diffusive properties of materials within the MOF-808 family provides unique opportunities for research into tailored MOF materials.

\section{CONCLUSIONS}

In this work, we have demonstrated that MOF-808-acetate provides a better starting point for many applications than the more commonly described MOF-808-formate. The MOF-808acetate is more tolerable to heating, where it retains its high surface area after exposure to higher temperatures than the formate-based material. We also introduce the novel MOF808-benzoate, which can easily be synthesized from both the acetate and the formate analogues. The introduction of the benzoate ligands yields a thermally stable MOF, such as the MOF-808-acetate. Benzoate incorporation also changes the gas-adsorptive behavior of the MOF. We believe that the opportunity to fine-tune the thermal stability and adsorptive behavior of these MOFs paves the way for optimizing their properties in a variety of applications including heterogeneous catalysis and separation technologies.

\section{ASSOCIATED CONTENT}

\section{s1 Supporting Information}

The Supporting Information is available free of charge at https://pubs.acs.org/doi/10.1021/acs.chemmater.0c04823.

Detailed experimental procedures, characterization (PXRD, ${ }^{1} \mathrm{H}$ NMR, TGA-DSC, $\mathrm{N}_{2}$-adsorption, scanning electron microscopy, and $\mathrm{CO}_{2}+$ hydrocarbon adsorption), and breakthrough experiments (PDF)

\section{AUTHOR INFORMATION}

\section{Corresponding Author}

Karl Petter Lillerud - Section for Catalysis, Department of Chemistry, University of Oslo, Oslo N-0315, Norway; ProfMOF A/S, Kongsberg 3616, Norway;

Email: k.p.lillerud@kjemi.uio.no

\section{Authors}

Erlend Aunan - Section for Catalysis, Department of Chemistry, University of Oslo, Oslo N-0315, Norway; (1) orcid.org/0000-0002-0589-7991

Christopher W. Affolter - ProfMOF A/S, Kongsberg 3616, Norway

Unni Olsbye - Section for Catalysis, Department of Chemistry, University of Oslo, Oslo N-0315, Norway; (1) orcid.org/0000-0003-3693-2857

Complete contact information is available at: https://pubs.acs.org/10.1021/acs.chemmater.0c04823 


\section{Author Contributions}

${ }^{\S}$ E.A. and C.W.A. contributed equally.

\section{Funding}

This project was funded by the Department of Chemistry and the Faculty of Mathematics and Natural Sciences at the University of Oslo. This work was partly supported by the Research Council of Norway through the Norwegian NMR Package in 1994 and partly supported by the Research Council of Norway through the Norwegian NMR Platform, NNP (226244/F50), as well as The Norwegian Centre for X-ray diffraction, Scattering and Imaging (RECX) (no. NFR 208896/F50).

\section{Notes}

The authors declare the following competing financial interest(s): K.P.L and C.W.A are affiliated with ProfMOF A/ $\mathrm{S}$, a private company seeking to commercialize MOF materials.

\section{ACKNOWLEDGMENTS}

The authors thank Dr. Evgeniy A. Redekop and Dr. Sigurd Øien-Ødegaard for invaluable discussions. We are also grateful for the consultation from Prof. Dr. Silvia Bordiga and Dr. Andrea Lazzarini for providing spectroscopic data.

\section{REFERENCES}

(1) Kalmutzki, M. J.; Hanikel, N.; Yaghi, O. M. Secondary Building Units as the Turning Point in the Development of the Reticular Chemistry of MOFs. Sci. Adv. 2018, 4, No. eaat 9180.

(2) Yaghi, O. M.; Kalmutzki, M. J.; Diercks, C. S. Emergence of Metal-Organic Frameworks. Introduction to Reticular Chemistry; Wiley-VCH Verlag GmbH, 2019; pp 3-4.

(3) Cavka, J. H.; Jakobsen, S.; Olsbye, U.; Guillou, N.; Lamberti, C.; Bordiga, S.; Lillerud, K. P. A New Zirconium Inorganic Building Brick Forming Metal Organic Frameworks with Exceptional Stability. J. Am. Chem. Soc. 2008, 130, 13850-13851.

(4) Liu, Y.; Klet, R. C.; Hupp, J. T.; Farha, O. Probing the Correlations between the Defects in Metal-Organic Frameworks and Their Catalytic Activity by an Epoxide Ring-Opening Reaction. Chem. Commun. 2016, 52, 7806-7809.

(5) Yang, D.; Gates, B. C. Catalysis by Metal Organic Frameworks: Perspective and Suggestions for Future Research. ACS Catal. 2019, 9, $1779-1798$.

(6) Bon, V.; Senkovska, I.; Baburin, I. A.; Kaskel, S. Zr- and HfBased Metal-Organic Frameworks: Tracking Down the Polymorphism. Cryst. Growth Des. 2013, 13, 1231-1237.

(7) Mondloch, J. E.; Bury, W.; Fairen-Jimenez, D.; Kwon, S.; DeMarco, E. J.; Weston, M. H.; Sarjeant, A. A.; Nguyen, S. T.; Stair, P. C.; Snurr, R. Q.; Farha, O. K.; Hupp, J. T. Vapor-Phase Metalation by Atomic Layer Deposition in a Metal-Organic Framework. J. Am. Chem. Soc. 2013, 135, 10294-10297.

(8) Furukawa, H.; Gándara, F.; Zhang, Y.-B.; Jiang, J.; Queen, W. L.; Hudson, M. R.; Yaghi, O. M. Water Adsorption in Porous MetalOrganic Frameworks and Related Materials. J. Am. Chem. Soc. 2014, 136, 4369-4381.

(9) Zhang, Y.; Zhang, X.; Lyu, J.; Otake, K.-i.; Wang, X.; Redfern, L. R.; Malliakas, C. D.; Li, Z.; Islamoglu, T.; Wang, B.; Farha, O. K. A Flexible Metal-Organic Framework with 4-Connected Zr6 Nodes. J. Am. Chem. Soc. 2018, 140, 11179-11183.

(10) Yang, Y.; Zhang, X.; Kanchanakungwankul, S.; Lu, Z.; Noh, H.; Syed, Z. H.; Farha, O. K.; Truhlar, D. G.; Hupp, J. T. Unexpected "Spontaneous" Evolution of Catalytic, MOF-Supported Single Cu(II) Cations to Catalytic, MOF-Supported $\mathrm{Cu}(0)$ Nanoparticles. J. Am. Chem. Soc. 2020, 142, 21169-21177.

(11) Bai, Y.; Dou, Y.; Xie, L.-H.; Rutledge, W.; Li, J.-R.; Zhou, H.-C. Zr-Based Metal-Organic Frameworks: Design, Synthesis, Structure, and Applications. Chem. Soc. Rev. 2016, 45, 2327-2367.
(12) Yaghi, O. M.; Kalmutzki, M. J.; Diercks, C. S. Binary MetalOrganic Frameworks. Introduction to Reticular Chemistry; Wiley-VCH Verlag GmbH, 2019; pp 95-96.

(13) Baek, J.; Rungtaweevoranit, B.; Pei, X.; Park, M.; Fakra, S. C.; Liu, Y.-S.; Matheu, R.; Alshmimri, S. A.; Alshehri, S.; Trickett, C. A.; Somorjai, G. A.; Yaghi, O. M. Bioinspired Metal-Organic Framework Catalysts for Selective Methane Oxidation to Methanol. J. Am. Chem. Soc. 2018, 140, 18208-18216.

(14) Meng, X.; Wang, H.-N.; Wang, L.-S.; Zou, Y.-H.; Zhou, Z.-Y. Enhanced Proton Conductivity of a MOF-808 Framework through Anchoring Organic Acids to the Zirconium Clusters by Post-Synthetic Modification. CrystEngComm 2019, 21, 3146-3150.

(15) Jiang, J.; Gándara, F.; Zhang, Y.-B.; Na, K.; Yaghi, O. M.; Klemperer, W. G. Superacidity in Sulfated Metal-Organic Framework-808. J. Am. Chem. Soc. 2014, 136, 12844-12847.

(16) Lin, Z.-J.; Zheng, H.-Q.; Zeng, Y.-N.; Wang, Y.-L.; Chen, J.; Cao, G.-J.; Gu, J.-F.; Chen, B. Effective and Selective Adsorption of Organoarsenic Acids from Water over a Zr-Based Metal-Organic Framework. Chem. Eng. J. 2019, 378, 122196.

(17) Cho, K. H.; Chitale, S. K.; Kim, S.-J.; Cha, G.-Y.; Hong, D.-Y.; Ryu, S. G.; Chang, J.-S.; Hwang, Y. K. Adsorptive Removal of NerveAgent Simulant with Zirconium-Based Metal-Organic Frameworks Modified by Hydrophobic Monocarboxylic Acids. Microporous Mesoporous Mater. 2019, 285, 61-69.

(18) Peng, Y.; Huang, H.; Zhang, Y.; Kang, C.; Chen, S.; Song, L.; Liu, D.; Zhong, C. A Versatile MOF-Based Trap for Heavy Metal Ion Capture and Dispersion. Nat. Commun. 2018, 9, 187.

(19) Liu, Q.; Zhang, Q.; Liu, B.; Ma, J. A New Synthesis and Adsorption Mechanism of $\mathrm{ZrO}_{2}$ Based Metal-Organic Frames for Efficient Removal of Mercury Ions from Aqueous Solution. Ceram. Int. 2019, 45, 15720-15724.

(20) Moon, S.-Y.; Liu, Y.; Hupp, J. T.; Farha, O. K. Instantaneous Hydrolysis of Nerve-Agent Simulants with a Six-Connected Zirconium-Based Metal-Organic Framework. Angew. Chem., Int. Ed. 2015, 54, 6795-6799.

(21) Reinsch, H.; Waitschat, S.; Chavan, S. M.; Lillerud, K. P.; Stock, N. A Facile "Green" Route for Scalable Batch Production and Continuous Synthesis of Zirconium MOFs. Eur. J. Inorg. Chem. 2016, 2016, 4490-4498.

(22) It is important to note that all of the aforementioned gas sorption experiments were conducted using the same set of samples. There is a therefore a visible decline in MOF-808-formate's gas capacity, especially for the hydrocarbon samples. We believe that this decline is due to the material's repeated pre-treatments before each measurement. These pretreatment programs are necessary and involve high vacuum and relative high temperature $\left(150^{\circ} \mathrm{C}\right)$ for a prolonged period. The decline is associated with a loss of available surface area, resulting in reduced capacity. Despite the decline in capacity, we assume that the heat of adsorptions are not significantly affected, and that the experimental results provided in Table 1 are still valid.

(23) Liang, W.; Chevreau, H.; Ragon, F.; Southon, P. D.; Peterson, V. K.; D'Alessandro, D. M. Tuning Pore Size in a ZirconiumTricarboxylate Metal-Organic Framework. CrystEngComm 2014, 16, $6530-6533$. 